\title{
Reflex sympathetic dystrophy associated with squamous cell carcinoma of the lung
}

\author{
M PROWSE, ${ }^{1}$ C M B HIGGS, ${ }^{2}$ C FORRESTER-WOOD,$^{3}$ AND \\ N MCHUGH
}

From the ${ }^{1}$ Royal National Hospital for Rheumatic Diseases, Bath; the ${ }^{2}$ Royal United Hospital, Bath; and the ${ }^{3}$ Frenchay Hospital, Bristol

SUMMARY Reflex sympathetic dystrophy was the presenting feature in an otherwise occult case of non-metastatic squamous cell carcinoma of the lung which improved on surgical removal of the primary tumour. Reflex sympathetic dystrophy, therefore, should be considered an occasional manifestation of a paraneoplastic syndrome warranting a thorough search for underlying malignancy.

Reflex sympathetic dystrophy is a painful limb disorder characterised by vasomotor and sudomotor dysfunction, patchy osteoporosis, and, later, contracture formation. The cause is unknown, though it is the occasional accompaniment of soft tissue trauma, bony fractures, cerebrovascular accidents, peripheral nerve injuries, and certain drugs, including barbiturates. ${ }^{12}$ There have, however, been a number of case reports of this syndrome as a nonmetastatic manifestation of underlying carcinoma, ${ }^{3-6}$ suggesting the involvement of a circulating humoral factor(s). We report a case in association with a squamous cell carcinoma of the lung with a marked improvement in the clinical condition after successful surgical removal of this otherwise asymptomatic lesion.

\section{Case report}

The patient a 74 year old man, developed pain in the right arm three days after changing a car tyre. The pain was diffuse, affecting the entire right upper limb with shooting pains extending to each of the digits. The pain was continuous for several months, unrelieved by anti-inflammatory drugs, and was associated with tenderness, diffuse swelling of the limb, cyanotic colour changes, and a loss of function of the hand and wrist. He was otherwise well, had

Accepted for publication 6 August 1988.

Correspondence to Dr N McHugh, Royal National Hospital for Rheumatic Diseases, Upper Borough Walls, Bath BA1 1RL. no previous major illnesses, and was receiving no other treatments. He had been a non-smoker for 25 years, previously having smoked 40 cigarettes a day.

When examined four months after the onset of his pain he had evidence of global restriction of right shoulder movements. Elbow movements were full and pain free. There were 20 degrees of flexion and extension of the right wrist with a flexion deformity of metacarpophalangeal, proximal interphalangeal, and distal interphalangeal joints, giving rise to a claw hand. There was patchy cyanotic discoloration of the arm with erythema overlying the small joints of the hand and wasting of the forearm musculature. Reflexes were symmetrical and normal, and sensation was intact bilaterally.

An $x$ ray examination of the right forearm and hand showed patchy osteopenia (Fig. 1). Thermography showed temperature increase in the right forearm and hand. Dual photon absorptiometry demonstrated a markedly reduced bone mineral density in the right hand (linear mean (right) $0.97 \mathrm{~g}$ $\mathrm{HA} / \mathrm{cm}$, (left) $1.70 \mathrm{~g} \mathrm{HA} / \mathrm{cm}$ ) (Fig. 2).

Chest $x$ ray demonstrated a $4 \mathrm{~cm}$ diameter circumscribed opacity in the left upper lobe with no evidence of mediastinal lymphadenopathy (Fig. 3). Other investigations were haemoglobin $158 \mathrm{~g} / \mathrm{l}$, white blood cell count $7.3 \times 10^{9} / 1$, viscosity $2.81 \mathrm{cP}$, calcium $2.45 \mathrm{mmol} / 1$ (normal range 2.20-2.70), phosphate $0.89 \mathrm{mmol} / \mathrm{l}$ (normal range $0.8-1.5$ ), alkaline phosphatase marginally raised at $117 \mathrm{IU} / \mathrm{l}$ (normal range 21-92), liver function tests normal. A computed tomographic scan of the brain was normal. 
An $x$ ray of the cervical spine showed moderate cervical spondylosis. Bronchoscopy and computed tomographic scanning of lung and mediastinum were performed, which showed a localised lesion in the left upper lobe with no evidence of enlarged mediastinal nodes. The patient underwent left upper lobectomy for removal of the tumour and made an uneventful postoperative recovery. Histology de- monstrated a poorly differentiated squamous ce $\bar{P}$. carcinoma of the lung with no involvement of locat: or hilar nodes, or pleura, but tumour was demonstrated in perineural spaces.

On awakening from surgery the pain in the patient's right arm had disappeared, and he has remained pain free for three months. His flexio contractures have persisted but improved with seriai

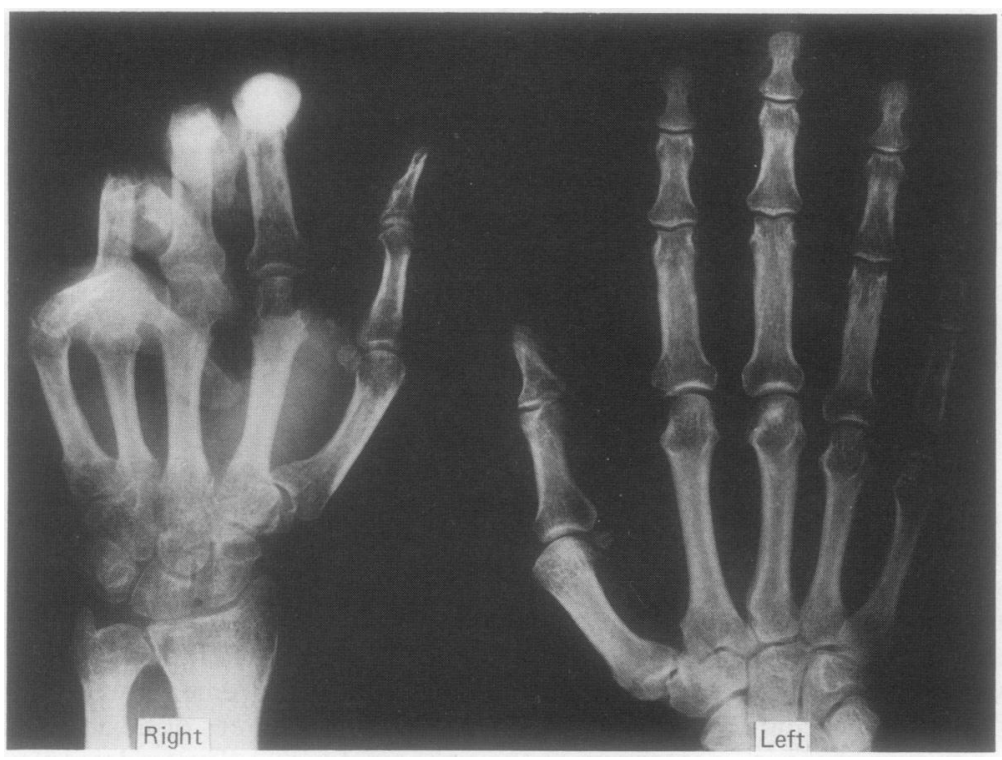

Fig. 1 Plain radiograph demonstrates patchy osteoporosis and contracture formation of the right hand.

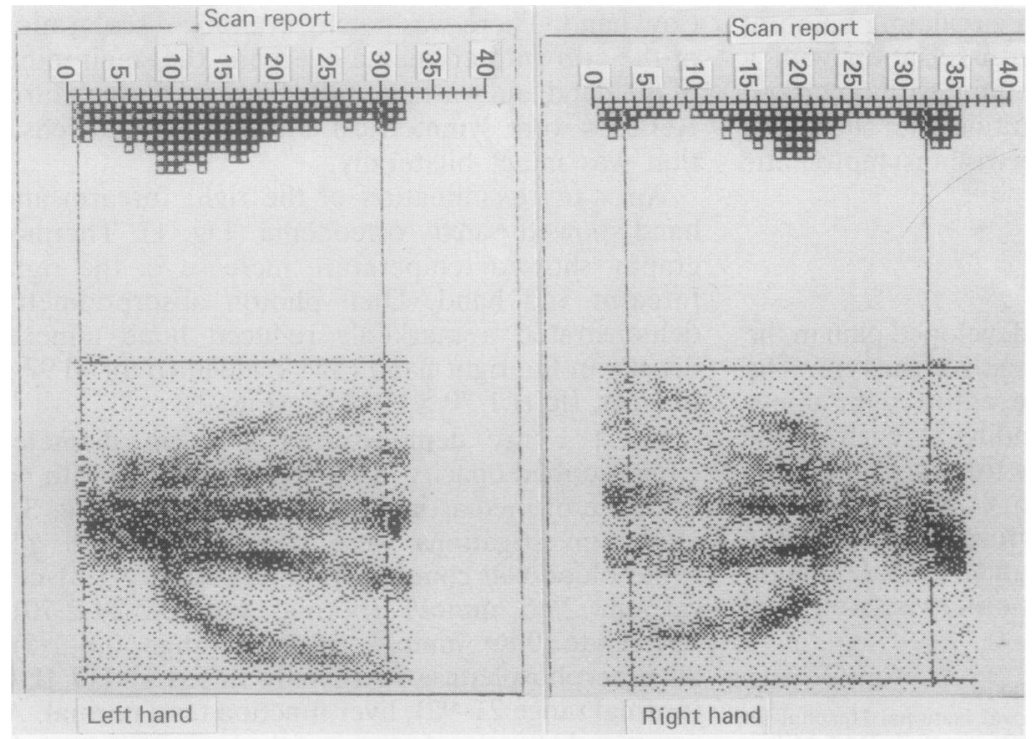

Fig. 2 Dual photon bone scan N demonstrates decreased bone mineral density of the right hando

$\vec{b}$

㐌




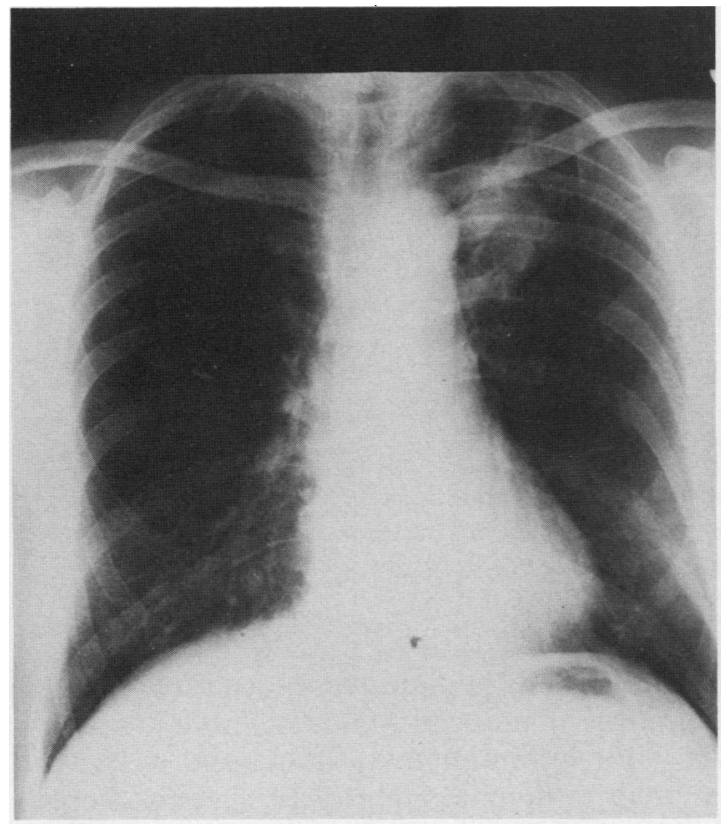

Fig. 3 Chest $x$ ray demonstrating the $4 \mathrm{~cm}$ diameter opacity in the left upper zone.

splinting and intensive physiotherapy. The global restriction of the right shoulder movements resolved after intra-articular injection of corticosteroid. A further $x$ ray of the hand has not changed, although dual photon bone scan shows a modest improvement in bone mineral content (linear mean (right) $1.04 \mathrm{~g} \mathrm{HA} / \mathrm{cm}$, (left) $1.80 \mathrm{~g} \mathrm{HA} / \mathrm{cm}$ ).

\section{Discussion}

This case highlights the association of reflex sympathetic dystrophy with underlying malignancy. The search for a cause of our patient's reflex sympathetic dystrophy enabled an otherwise asymptomatic lung tumour to be successfully removed at a relatively early stage. There have been a number of published reports of cases of the reflex sympathetic dystrophy syndrome $e^{3-6}$ in association with malignancy, including carcinoma of bowel, ovary, pancreas, lung, and chronic myelogenous leukaemia. In most of the cases reviewed the syndrome was the presenting feature with the tumour found on subsequent investigations, though often the association was tenuous with prolonged periods between development of the syndrome and presentation of the tumour. ${ }^{5}$

We have been unable to find any reports where the reflex sympathetic dystrophy syndrome reversed after successful tumour removal, which is important in establishing a direct relation between otherwise separate conditions. In our case we feel the association is more than coincidental because of the lack of resolution of the reflex sympathetic dystrophy with various treatments before the operation and the dramatic improvement in pain and vasomotor disturbances in the immediate postoperative period, though the contractures have been much slower to improve. Bremer did demonstrate transient improvement after ovarian tumour regression with radiotherapy, but the patient died two months after tumour diagnosis. ${ }^{7}$

Although the aetiology of reflex sympathetic dystrophy is unknown, its occasional association with an apparently distant neoplastic process suggests that as yet unidentified circulating humoral factors may be involved. It is possible that the tumour produces humoral factor(s) perhaps acting at either the internuncial pool of the spinal cord to lower fibre threshold with increased activity spreading to sympathetic efferents or at a peripheral site to stimulate sympathetic fibres. There is some evidence suggesting that noradrenaline lowers the threshold of peripheral mechanoreceptors, ${ }^{89}$ thereby enhancing abnormal firing in peripheral sensory nerves, which would account for the spontaneous pain and hyperaesthesia seen clinically.

The development of reflex sympathetic dystrophy as a non-metastatic manifestation enabled detection and successful removal of an otherwise asymptomatic lung carcinoma. The case highlights the need for a thorough search for underlying malignancy in patients presenting with 'idiopathic' reflex sympathetic dystrophy.

\section{References}

1 Schwartzman R J, McLellon T L. Reflex sympathetic dystrophy. A review. Arch Neurol 1987; 44: 555-61.

2 Schutzer S F, Gossling H R. The treatment of reflex sympathetic dystrophy. J Bone Joint Surg [Am] 1987; 66: 625-9.

3 Medsger T A Jr, Dixon J A, Garwood F. Palmar fasciitis and polyarthritis associated with ovarian carcinoma. Ann Intern Med 1982; 96: 428-31.

4 Baer A N, Phillips R M. Pancreatic carcinoma and palmar fasciitis. Ann Intern Med 1983; 99: 411-2.

5 Goldberg E, Dobransky R, Gill - R. Reflex sympathetic dystrophy associated with malignancy. Arthritis Rheum 1985; 28: $1079-80$.

6 Michaels R M, Sosber J A. Reflex sympathetic dystrophy as a probable paraneoplastic syndrome: case report and literature reviewed. Arthritis Rheum 1984; 27: 1183-5.

7 Bremer C. Shoulder-hand syndrome; a case of unusual aetiology. Annals of Physical Medicine 1966; 9: 168-71.

8 De Takats G. Causalgia status in peace and war. JAMA 1945; 78: 699-704.

9 Devor $M$. Nerve pathophysiology and mechanisms of pain in causalgia. J Auton Nerv Syst 1983; 7: 371-84. 